\title{
New Criterion for Borderline Resectable Pancreatic Cancer: Invasion of a Replaced Right Hepatic Artery
}

\author{
Florin Botea ${ }^{1}$, Traian Dumitrascu ${ }^{1}$, Dragos Zamfirescu ${ }^{2}$, Alexandru Onofrei ${ }^{1}$, Diana Nicolaescu ${ }^{1}$, \\ Alexandru Barcu ${ }^{1}$, Ruxandra Fota ${ }^{3}$, Irinel Popescu ${ }^{1}$
}

"Dan Setlacec" Center of General Surgery and Liver Transplantation, Fundeni Clinical Institute, Bucharest, Romania

2Department of plastic and reconstructive surgery, "Zetta" Clinic, Bucharest

${ }^{3}$ Intensive Care Unit, Fundeni Clinical Institute, Bucharest, Romania
Corresponding author:

Irinel Popescu, MD, PhD, FACS, FEBS

Center of General Surgery and Liver

Transplantation, Fundeni Clinical

Institute, Fundeni Street no. 258,

022328, Bucharest, Romania

E-mail: irinel.popescu220@gmail.com

\section{ABSTRACT}

Background: Loco-regional invasion is not uncommon in a patient diagnosed with a pancreatic cancer. A negative resection margins pancreatectomy represents the most important determining factor of survival in patients resected for pancreatic adenocarcinoma. Thus, in order to increase resectability in such patients, extended pancreatectomies were proposed in so-called borderline resectable tumors, including venous, arterial or other surrounding organs removal.

Case presentation: A 52-year old male, with pancreatic head cancer invasive into the portal vein and replaced right hepatic artery (rRHA)(from superior mesenteric artery), underwent pancreatico-duodenectomy (PD) with portal vein resection and reconstruction, segmental resection of the rRHA and associated right hemi-hepatectomy.

Results: The postoperative outcome was remarkably uneventful except for a self-limited minor bile leak. Negative resection margins of the operative specimen were observed at pathological examination.

Conclusion: Proper assessment of the presence and course of a rRHA prior to PD is mandatory for a safe surgery. Invasion of the rRHA is not a contraindication for resection in a patient with pancreatic head cancer and should be included among the borderline resection criteria. Associated right hemi-hepatectomy is a safe option for such a patient in order to achieve negative resection margins.

Key words: pancreatic cancer, replaced right hepatic artery, pancreatico-dudenectomy, hemihepatectomy

\section{INTRODUCTION}

Pancreatic adenocarcinoma (PA) is an important healthcare problem worldwide: the fourth leading cause of cancer related death in the United States (1) and the fifth leading cause of cancer related death in Europe (2). 
Surgery (i.e. resection) represents the only chance for a long-term survival, but over $80 \%$ of the patients with PA are diagnosed when unresectable (3). Moreover, studies have shown that survival rates are significantly higher in patients with locally-advanced PA and resection, when compared with the non-resected patients $(4,5)$. Nevertheless, a negative resection margins pancreatectomy represents the most important determinant of the survival in patients resected for PA (6). Loco-regional invasion is not uncommon in patients with PA (1). In order to increase resectability in such patients, extended pancreatectomies were proposed, including venous, arterial or other surrounding organs removal (5). The borderline resection criteria for selecting these patients include the involvement of portal and superior mesenteric veins, and/or hepatic, superior mesenteric and celiac arteries.

Portal vein invasion no longer contraindicates resection in patients with PA if the venous reconstruction is technically feasible $(5,7)$. However, an increased morbidity should be expected if venous resection is performed, compared with the patients without a portal vein resection (8). Furthermore, patients with a histological proof of venous invasion have significantly worse survival rates, compared with the patients without portal vein resection (8), but much better compared with the non-resected patients (5).

Arterial resections in a patient with PA have been demonstrated to be technically feasible and safe $(9,10)$. Arterial resections during pancreatectomies are considered controversial in terms of benefits because of very high morbidity and mortality rates, mainly associated with the arterial reconstruction (11). However, recent studies have shown potential survival benefits for pancreatectomies with arterial resection for PA (12).

A replaced right hepatic artery ( $\mathrm{rHHA}$ ) arising from the superior mesenteric artery (SMA) is a relatively frequent anatomical variant (13) and might change the surgical strategy in patients submitted for pancreaticoduodenectomy (PD). Thus, a posterior approach might be useful to avoid the rRHA injuries during PD (14). However, in patients with PA invading the rRHA, in order to achieve negative resection margins, arterial resection is mandatory. Resection of the $\mathrm{RHHA}$ during PD has the potential to increase the morbidity rate and to determine liver ischemia, necrosis and sepsis.

Hereby, are presented the surgical strategy and outcomes of a patient with PA of the pancreatic head with portal vein and rRHA invasion.

\section{CASE REPORT}

A 52-year old male, with a history of chronic hepatitis $\mathrm{C}$, presented a two months history of jaundice and weight loss (6 kg). The blood analysis showed cholestasis (total bilirubin $=10 \mathrm{mg} / \mathrm{L}$ ) and slight cytolysis (ALAT= $158 \mathrm{U} / \mathrm{l} ; \mathrm{ASAT}=106 \mathrm{U} / \mathrm{l})$; the serum carbohydrate antigen level (CA 19-9) was elevated $(369.7 \mathrm{U} / \mathrm{mL})$, and the carcinoembryonic antigen (CEA) was normal (2.1 $\mathrm{ng} / \mathrm{mL}$ ).

Ultrasound, endoscopic ultrasound, cholangiomagnetic resonance (fig. 1) and abdominal contrast enhanced computed tomography (fig. 2), showed a 2/2 $\mathrm{cm}$ cephalic pancreatic tumor, dilated intrahepatic and extrahepatic bile ducts (diameter of $23 \mathrm{~mm}$ ), dilated Wirsung (diameter of 3-4 $\mathrm{mm}$ ), arterial anatomical variation - rRHA ( $2.5 \mathrm{~mm}$ in caliber) originating from the SMA, enlarged (non-tumoral) celiac, inter-aorto-caval and hepatic hilum lymph nodes. The tumor was very close to the portal vein and was invasive into the rRHA (fig. 2). No distant metastases were observed at the preoperative imaging examinations. The tumor was initially considered as unresectable in another unit. Therefore, a biliary stent was placed as palliative jaundice treatment, which was functional for only two weeks, and was consequently replaced, but without jaundice remittance. For this reason, the patient was referred to our center. Liver volumetry showed that the right hemi-liver represented $50 \%$ of the total liver volume.

The patient was discussed at the oncology multidisciplinary board and considered to have a borderline resectable PA, and was therefore scheduled for surgery.

The intraoperative diagnosis was pancreatic head

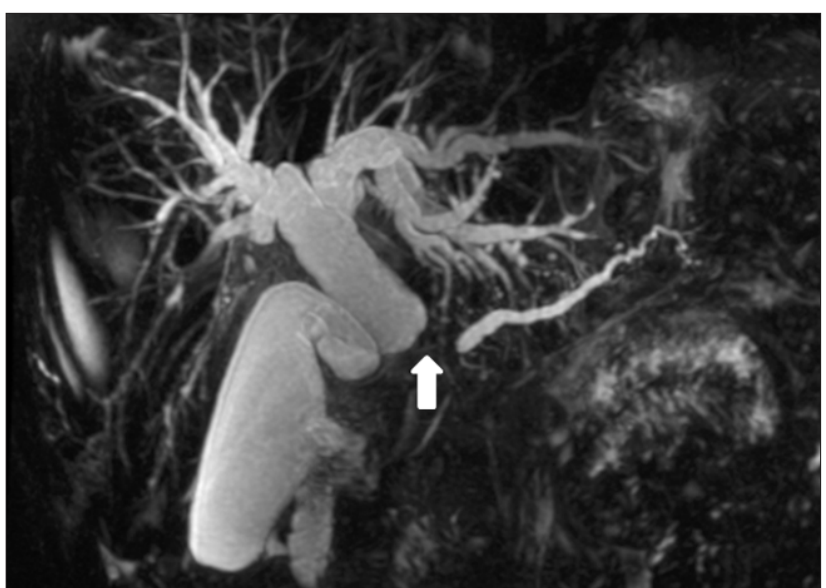

Figure 1 - Cholangio-magnetic resonance showing dilated intrahepatic and extrahepatic bile ducts, along with an enlarged Wirsung duct, both latter ones ending in an abrupt manner ("double duct sign" - white arrow) 


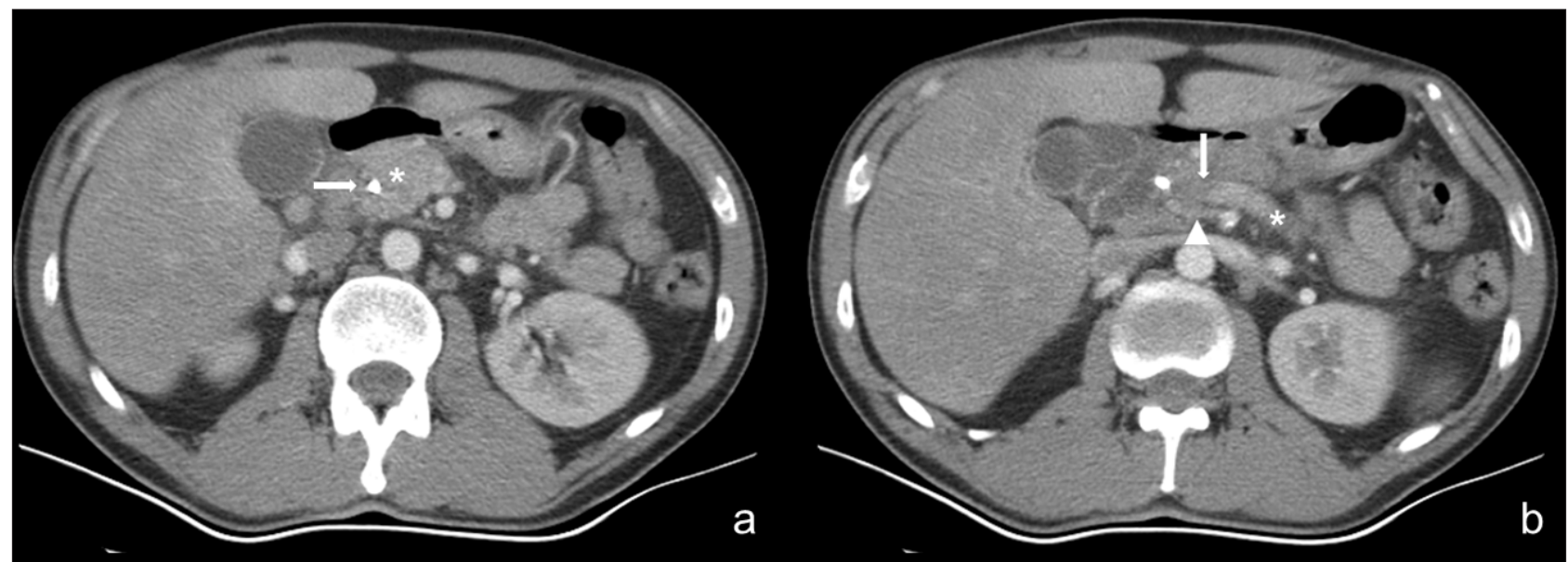

Figure 2 - Preoperative abdominal contrast enhanced computed tomography showing: a. the pancreatic head tumor $\left({ }^{*}\right)$ and the biliary stent (arrow); b. portal vein (arrow) and replaced right hepatic artery (arrowhead) in close contact with the tumor

tumor invading the distal bile duct, the rRHA and the portal vein. Consequently, a Whipple PD was performed associated with segmental portal resection, segmental resection of the $\mathrm{rRHA}$ (at $2 \mathrm{~cm}$ after its origin) and consecutive right hemi-hepatectomy (fig. 3). The hepatectomy was performed using the anterior approach and intraoperative ultrasound guidance, in order to preserve as much viable

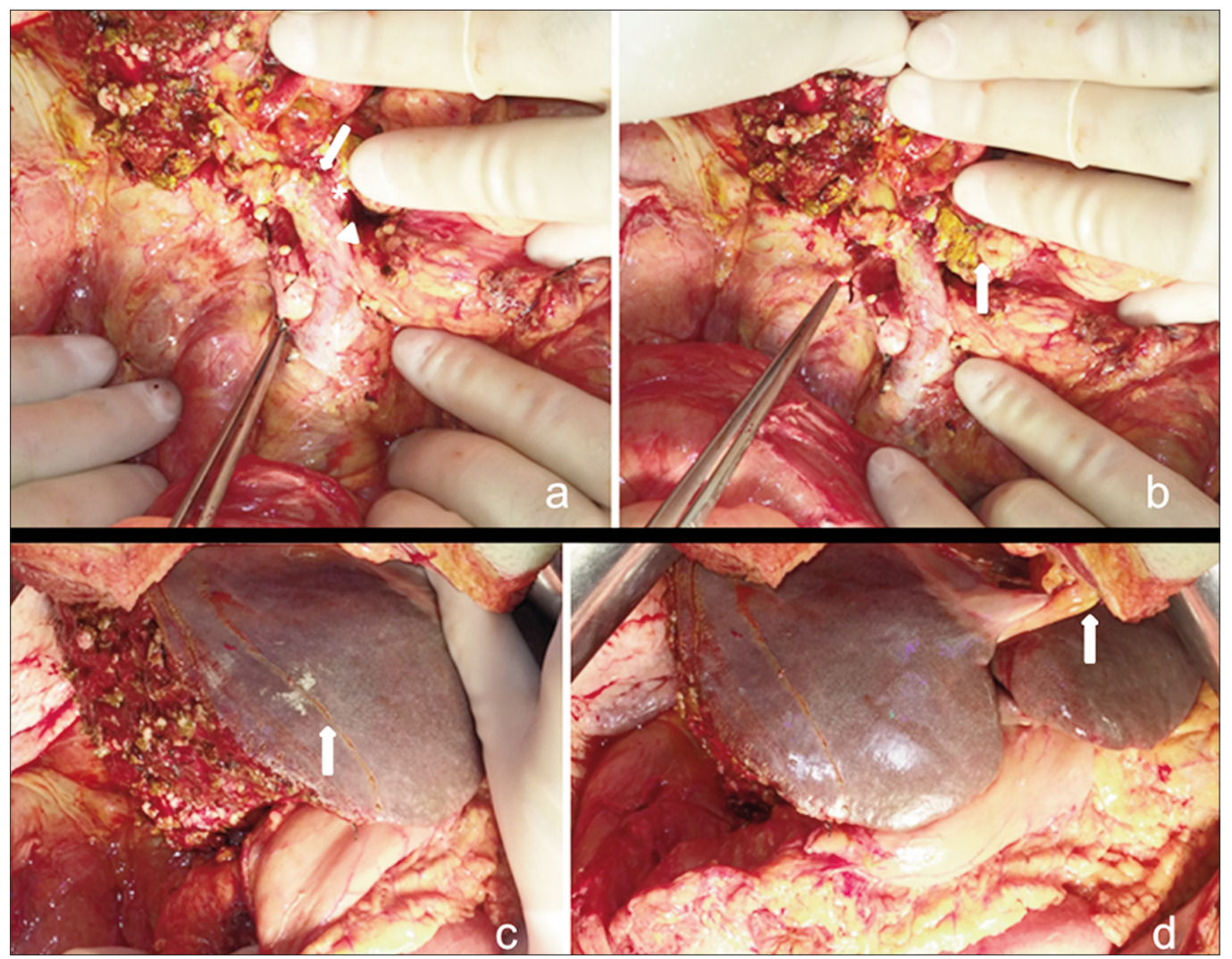

Figure 3 - Intraoperative aspects after pancreatico-duodenectomy with segmental resection of the portal vein with end-to-end anastomosis, segmental resection of the replaced right hepatic artery and right hemi-hepatectomy:

a) proximal stump of the replaced right hepatic artery showed by the tip of the clamp, end-to-end portal anastomosis (arrow), inferior mesenteric vein (arrow head), and splenic vein (*); b) distal stump of the replaced right hepatic artery (indicated by the tip of the clamp), pancreatic stump (arrow); c) remnant liver and cut surface area after

right hemi-hepatectomy; note the difference between the cut surface and the Cantlie's line (arrow); d) remnant liver attached to the anterior abdominal wall using the falciform ligament (arrow), in order to avoid torsion of the hepatic veins 


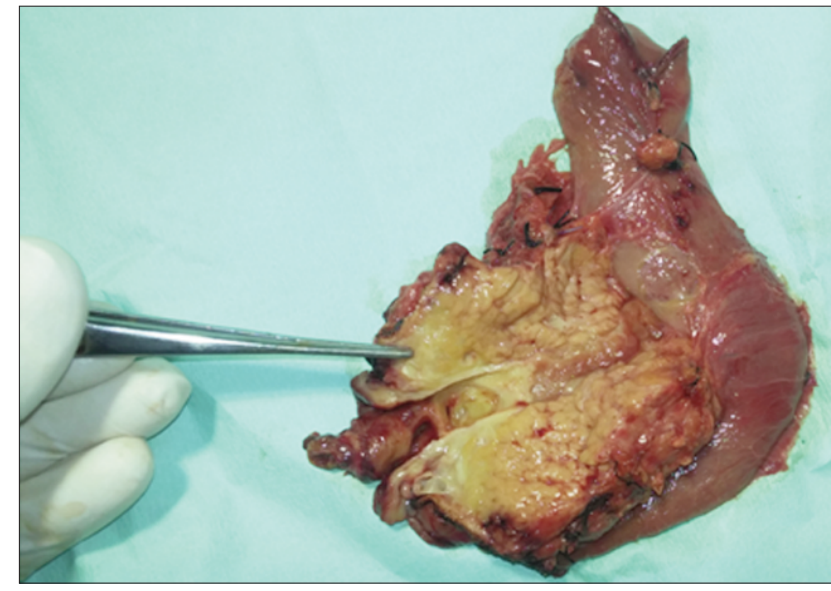

Figure 4 - Pancreatico-duodenectomy for pancreatic head adenocarcinoma (indicated by the tip of the clamp)

remnant liver as possible (fig. 3). The portal reconstruction was performed in an end-to-end tension-free fashion, between the distal portal stump and the confluence of the inferior mesenteric and splenic veins. Graft inter-positioning was avoided by sectioning the right portal vein (step included in the hemihepatectomy technique), thus obtaining the elongation of the distal portal stump. Furthermore, the reconstruction continued with an end-to-side Wirsungo-jejunal duct-to-mucosa anastomosis (protected by an internal stent), end-to-side hepatico-jejunal anastomosis, and end-to-side gastro-jejunal anastomosis. The operative time was $540 \mathrm{~min}$. The intraoperative blood loss was approximately $800 \mathrm{ml}$; one unit of blood was transfused.

The pathological examination of the operative specimen (fig. 4) showed a well-differentiated pancreatic ductal adenocarcinoma with one retro- pancreatic lymph node metastasis - pT3, pN1, cM0, V1, A1 (stage IIbTNM); resection margins were considered as negative.

The postoperative outcome was remarkably uneventful except for a minor bile leak, remitted after conservative treatment. On the postoperative day 12 , a contrast enhanced computed tomography was performed, which showed no significant abnormalities (fig. 5).

The patient was discharged on the postoperative day 20 (the prolonged hospitalization was required for the conservative treatment of the bile leak), with regular 3-months follow-up (normal CA19-9,CEA and abdominal ultrasound). The patient currently undergoes adjuvant chemotherapy (gemcitabine and oxaliplatin -4 series).

\section{DISCUSSION}

Approximately one third of the patients diagnosed with PA have a regional-only disease (i.e., tumors with loco-regional invasion into the main vessels, without distant metastases). A subgroup of these patients is suitable for resection (1), and is classified as borderline resectable according to the criteria defined by the International Study Group of Pancreatic Surgery, based on the involvement of the surrounding main vessels (7). However, anatomical variants, such as rRHA arising from SMA, were not taken into consideration; in our opinion, this presentation should also be defined as a borderline resectable criterion.

A borderline resectable PA involves an extended pancreatectomy in order to achieve negative resection margins, as it was the case in the above presented patient, where the PD was extended to portal vein

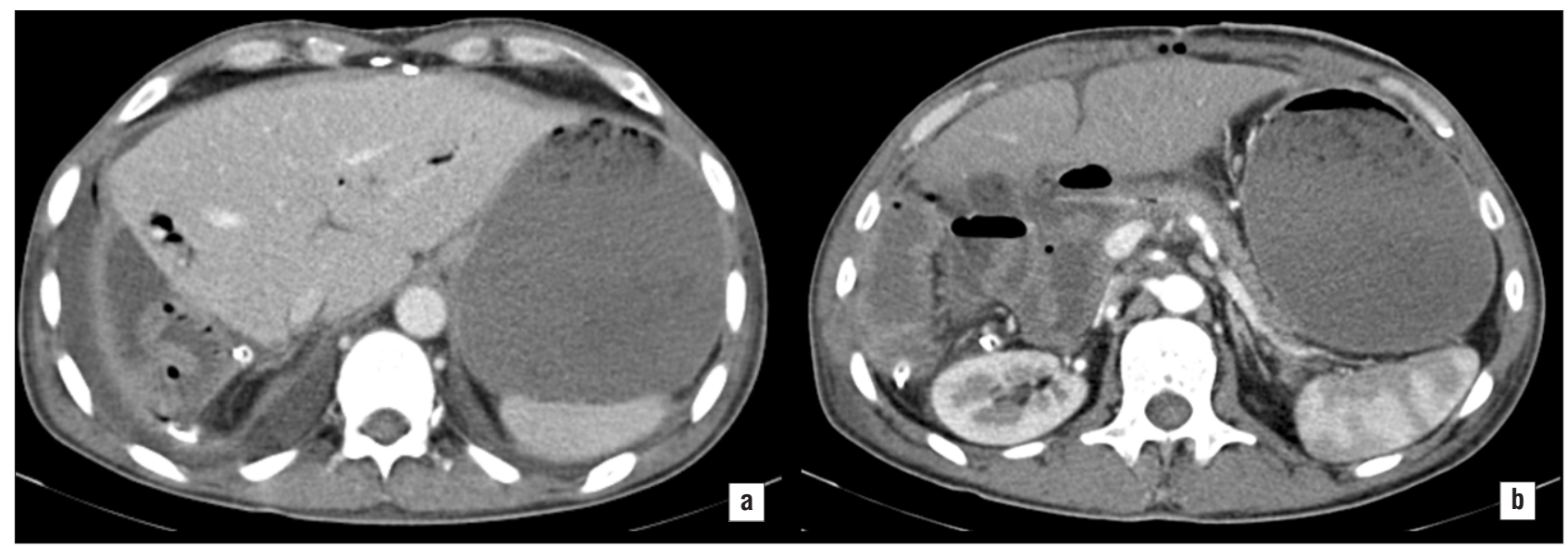

Figure 5. Postoperative abdominal contrast enhanced computed tomography showing the well-vascularized remnant liver (a) and regular remnant pancreas (b) 
resection and reconstruction, and to rRHA resection associated with right hemi-hepatectomy.

An extended pancreatectomy is widely accepted to have an increased morbidity rate, compared with the standard pancreatectomies, but the long-term survival rates are comparable (4). However, there are also studies that have shown no increased morbidity rates after an extended pancreatectomy, compared with the standard pancreatectomy, but extended pancreatectomies for PA were associated with worse survivals (15).

Currently, there is no consensus regarding the appropriate management of patients with borderline resectable PA. Thus, some surgical teams prefer an up-front surgery (16), while others start with a neoadjuvant treatment (17). The International Study Group for Pancreatic Surgery does not support the routine neoadjuvant therapy for borderline PA, if technical options of resections are possible (7). However, a recent meta-analysis has suggested that patients with borderline PA should be included in neoadjuvant protocols (18). In the above presented patient, a neoadjuvant treatment was not feasible because the jaundice could not be released by the endoscopic stent mount.

Regarding the impact of the portal vein resection associated with pancreatectomies for PA, a recent meta-analysis has shown increased mortality rates and worse survivals, compared with pancreatectomies without portal vein resection (19).

Some studies have shown that an accessory or rRHA can be encountered in up to $20 \%$ of patients during PD $(20,21,22)$, similar to the general population according Michels' classification (Type III) (23). Special care must be taken in detecting such arterial anomaly, as it can pass undetected at preoperative imaging in 33\% to $64 \%$ of cases $(24,25)$. According to the course of the rRHA in relation to the pancreas, there are three types: type I (postero-lateral to the pancreatic head), type II (intrapancreatic) and type III (within the superior mesenteric vein groove) (20). In the above presented patient, the rRHA could be classified as type I (invaded by the pancreatic tumor). The course of rRHA in patients scheduled for PD should be properly assessed by the preoperative multidetector row computed tomography examination (26), because inadvertent division of rRHA leads to ischemic lesions of the right hemiliver and the proximal bile duct (which receives its blood supply almost entirely from the RHA), with subsequent necrotic and septic complications.

Invaded rRHA is encountered in $2.7 \%$ to $20.7 \%$ of patients with PA scheduled for PD. $(22,27)$ Resection of rRHA resection during PD has been previously documented in a limited number of patients $(21,24$,
$25,28,29,30,31)$. In order to avoid ischemic liver injuries, most of the surgical teams prefer arterial reconstruction after rRHA resection during PD (21, $24,25,28)$. To date, there is no consensus about the cut off caliber from which rRHA should be repaired. Short segments of hepatic artery encased by tumor have been successfully resected and reconstructed by either primary anastomosis or reversed saphenous vein interposition graft (32). Another technical option is to anastomose the distal stump of the resected rRHA to the stump of the gastroduodenal artery, to the splenic artery (in an end-to-end or end-to-side fashion) using a saphenous vein jump graft (27), or to the middle colic artery (33). Another option for vein graft could be the gonadal vein (34). There is no data available on the complication rate after $\mathrm{rHH}$ reconstruction. However, it is known that the complication rate in microsurgical arterial reconstruction using interposed venous graft varies between $3 \%$ and $8 \%$. Moreover, hepatic artery with caliber less than $3 \mathrm{~mm}$ is a factor related to significantly higher complication rate in living donor liver transplantation (35); the rRHA in the presented case had a maximum caliber of $2.5 \mathrm{~mm}$.

Embolisation of the rRHA prior to PD is an interesting, safe and effective alternative for preventing liver ischemic injuries in such cases, but the limited experience does not recommend it as a standard procedure $(29,30)$. Ligation of the rRHA during PD has been also reported (24), but the risk of postoperative liver ischemic injuries is high.

To the best of our knowledge, the above reported patient is the first one in the literature with a combined PD and right hemi-hepatectomy for a PA invasive into the rRHA. The feasibility and safety of the combined pancreatectomy and hepatectomy has been previously demonstrated (36), but there is a limited number of patients reported in the literature $(36,37)$. Simultaneous liver resection and PD for oncologic HPB diseases is a procedure that is rarely performed, with only $1 \%$ of HBP cases in the USA, less than 400 cases reported from Asia, and about 100 cases reported in Europe (37). However, a recent study has shown that patients with synchronous PD and hemi-hepatectomy may have very high morbidity and in-hospital mortality rates $(87.5 \%$ and $18.2 \%$, respectively) (37). Nevertheless, the morbidity and mortality rates after combined pancreatectomies and hepatectomies appear to be lower, compared with pancreatectomies with arterial resection and reconstruction (mortality $0-18.2 \%$ vs. $0-45.5 \%$; morbidity $3-87.5 \%$ vs. 16.7 $100 \%)(11,36,37)$. This latter data, in addition to liver 
volumetry, were taken into consideration in the above presented patient, when a right hemi-hepatectomy was considered instead of an arterial reconstruction. Moreover, the length of the resected portion of the rRHA and the small vessel diameter (fig. $3 a$ and $b$ ), involving elaborate microsurgical reconstruction with high risk of complications, were further factors that were taken into consideration when an arterial reconstruction was ruled out in this case.

A fact that is worth mentioning is that the right hemi-hepatectomy (by dividing the right portal vein) favored a tension-free portal vein reconstruction without graft inter-positioning, which decreased the risk of intraoperative complications and postoperative morbidity. So, in this particular case, if rRHA reconstruction would have been performed, portal vein reconstruction using interposed graft would have had to be carried out, too; this scenario involved a high risk of complications, and was therefore avoided.

\section{CONCLUSION}

Proper assessment of the presence and course of a rRHA prior to PD is mandatory for safe surgery. Invasion of the rRHA is not a contraindication for resection in a patient with PA of the pancreatic head, and should be included among the borderline resection criteria. Associated right hemi-hepatectomy is a safe option for such a patient in order to achieve negative resection margins.

\section{REFERENCES}

1. Siegel RL, Miller KD, Jemal A. Cancer statistics, 2015. CA Cancer J Clin 2015 Jan;65(1):5-29.

2. Ferlay J, Steliarova-Foucher E, Lortet-Tieulent J, Rosso S, Coebergh $\mathrm{JW}$, Comber $\mathrm{H}$, et al. Cancer incidence and mortality patterns in Europe: estimates for 40 countries in 2012. Eur J Cancer 2013 Apr; 49(6):1374-403.

3. Li D, Xie K, Wolff R, Abbruzzese JL. Pancreatic cancer. Lancet 2004 Mar 27:363(9414):1049-57.

4. Hartwig W, Vollmer CM, Fingerhut A, Yeo CJ, Neoptolemos JP, Adham $\mathrm{M}$, et al. Extended pancreatectomy in pancreatic ductal adenocarcinoma: definition and consensus of the International Study Group for Pancreatic Surgery (ISGPS). Surgery 2014 Jul:156(1):1-14.

5. Konstantinidis IT, Warshaw AL, Allen JN, Blaszkowsky LS, Castillo CF Deshpande V, et al. Pancreatic ductal adenocarcinoma: is there a survival difference for $\mathrm{R} 1$ resections versus locally advanced unresectable tumors? What is a "true" R0 resection? Ann Surg 2013 Apr;257(4): 731-6.

6. Esposito I, Kleeff J, Bergmann F, Reiser C, Herpel E, Friess H, et al. Most pancreatic cancer resections are R1 resections. Ann Surg Onco 2008 Jun;15(6):1651-60

7. Bockhorn M, Uzunoglu FG, Adham M, Imrie C, Milicevic M, Sandberg $A A$, et al. Borderline resectable pancreatic cancer: a consensus statement by the International Study Group of Pancreatic Surgery (ISGPS). Surgery 2014 Jun;155(6):977-88.

8. Dumitrascu T, Dima S, Brasoveanu V, Stroescu C, Herlea V, Moldovan $\mathrm{S}$, et al. Impact of a portal/superior mesenteric vein resection during pancreatico-duodenectomy for pancreatic head adenocarcinoma. Minerva Chir 2014 Dec;69(6):301-13

9. Brasoveanu V, Dumitrascu T, Bacalbasa N, Zamfir R. Splenic artery used for replaced common hepatic artery reconstruction during pancreatoduodenectomy - a case report. Chirurgia (Bucur). $2009 \mathrm{Jul}$; 104(4):499-504.

10. Brasoveanu V, Anghel C, Barbu I, Pautov M, Ionescu MI, Motthor M, et al. Pancreatoduodenectomy en bloc with portal and superior mesenteric artery resection - a case report and literature review. Anticancer Res 2015 Mar;35(3):1613-8.

11. Mollberg N, Rahbari NN, Koch M, Hartwig W, Hoeger Y, Buchler MW, et al. Arterial resection during pancreatectomy for pancreatic cancer: a systematic review and meta-analysis. Ann Surg 2011 Dec;254(6):882-

12. Bachellier P, Rosso E, Lucescu I, Oussoultzoglou E, Tracey J, Pessaux $P$, et al. Is the need for an arterial resection a contraindication to pancreatic resection for locally advanced pancreatic adenocarcinoma? A case-matched controlled study. J Surg Oncol 2011 Jan 1;103(1):7584

13. Yang SH, Yin YH, Jang JY, Lee SE, Chung JW, Suh KS, et al. Assessment of hepatic arterial anatomy in keeping with preservation of the vasculature while performing pancreatoduodenectomy: an opinion. World J Surg 2007 Dec;31(12):2384-91.

14. Dumitrascu T, David L, Popescu I. Posterior versus standard approach in pancreatoduodenectomy: a case-match study. Langenbecks Arch Surg 2010 Aug;:395(6):677-84.

15. De Reuver PR, Mittal A, Neale M, Gill AJ, Samra JS. Extended pancreatoduodenectomy as defined by the International Study Group for Pancreatic Surgery is associated with worse survival but not with increased morbidity. Surgery $2015 \mathrm{Jul} ; 158(1): 183-90$.

16. Kelly KJ, Winslow E, Kooby D, Lad NL, Parikh AA, Scoggins CR, et al. Vein involvement during pancreaticoduodenectomy: is there a need for redefinition of "borderline resectable disease"? J Gastrointest Surg $2013 \mathrm{Jul} ; 17(7): 1209-17$

17. Rashid OM, Pimiento JM, Gamenthaler AW, Nguyen P, Ha TT, Hutchinson T, et al. Outcomes of a Clinical Pathway for Borderline Resectable Pancreatic Cancer. Ann Surg Oncol. 2016 Apr;23(4): 1371-9.

18. Tang K, Lu W, Qin W, Wu Y. Neoadjuvant therapy for patients with borderline resectable pancreatic cancer: A systematic review and meta-analysis of response and resection percentages. Pancreatology 2015 ii: S1424-3903(15)00712-7.

19. Giovinazzo F, Turri G, Katz MH, Heaton N, Ahmed I. Meta-analysis of benefits of portal-superior mesenteric vein resection in pancreatic resection for ductal adenocarcinoma. Br J Surg 2016 Feb;103(3): 179-91.

20. Jah A, Jamieson N, Huguet E, Praseedom R. The implications of the presence of an aberrant right hepatic artery in patients undergoing a pancreaticoduodenectomy. Surg Today 2009;39(8):669-74.

21. Rammohan A, Palaniappan R, Pitchaimuthu A, Rajendran K, Perumal SK, Balaraman K, et al. Implications of the presence of an aberrant right hepatic artery in patients undergoing pancreaticoduodenectomy. World J Gastrointest Surg 2014 Jan 27:6(1):9-13.

22. Kim PT, Temple S, Atenafu EG, Cleary SP, Moulton CA, McGilvray ID, et al. Aberrant right hepatic artery in pancreaticoduodenectomy for adeno-carcinoma: impact on resectability and post-operative outcomes. HPB (Oxford). 2014 Mar:16(3):204-11.

23. Michels NA. Newer anatomy of the liver and its variant blood supply and collateral circulation. Am J Surg 1966;112:337-47.

24. Turrini 0 , Wiebke EA, Delpero JR, Viret F, Lillemoe KD, Schmidt CM. Preservation of replaced or accessory right hepatic artery during pancreaticoduodenectomy for adenocarcinoma: impact on margin status and survival. J Gastrointest Surg 2010 Nov;14(11): 1813-9.

25. Stauffer JA, Bridges MD, Turan N, Nguyen JH, Martin JK. Aberrant right hepatic arterial anatomy and pancreaticoduodenectomy: recognition, prevalence and management. HPB (0xford) 2009 Mar;11(2): 161-5.

26. Staskiewicz G, Torres K, Denisow M, Torres A, Czekajska-Chehab E, Drop A. Clinically relevant anatomical parameters of the replaced right hepatic artery (RRHA). Surg Radiol Anat 2015 Dec;37(10):1225-31. 
27. Sulpice L, Rayar M, Paquet C, Bergeat D, Merdrignac A, Cunin D, et al Does an aberrant right hepatic artery really influence the short - and long-term results of a pancreaticoduodenectomy for malignant disease? A matched case-controlled study. J Surg Res. 2013 Dec; 185(2):620-5.

28. Allendorf JD, Bellemare S. Reconstruction of the replaced right hepatic artery at the time of pancreaticoduodenectomy. J Gastrointest Surg 2009 Mar;13(3):555-7.

29. Cloyd JM, Chandra V, Louie JD, Rao S, Visser BC. Preoperative embolization of replaced right hepatic artery prior to pancreaticoduodenectomy. J Surg Oncol 2012 Sep 15;106(4):509-12.

30. El AM, Leteurtre E, Sergent G, Ernst O, Maunoury V, Branche J, et al. Pancreatic head carcinoma and right hepatic artery: embolization management - A case report. J Gastrointest Oncol 2014 Aug;5(4): E80-E83.

31. Amano H, Miura F, Toyota N, Wada K, Katoh K, Hayano K, et al. Is pancreatectomy with arterial reconstruction a safe and useful procedure for locally advanced pancreatic cancer? J Hepatobiliary Pancreat Surg. 2009; 16(6):850-7.

32. Bold RJ, Charnsangavej C, Cleary KR, Jennings M, Madray A, Leach SD, et al. Major vascular resection as part of pancreaticoduodenec- tomy for cancer: radiologic, intraoperative, and pathologic analysis. J Gastrointest Surg. 1999 May-Jun;3(3):233-43.

33. Sekido H, Shimada H, Nakano A, Endo I, Fujii S. Hepatectomy and pylorus-preserving pancreatoduodenectomy with reconstruction of the hepatic artery, using the middle colic artery, for bile duct carcinoma: A case report. J Hep Bil Pancr Surg 1994; 1(2):200-203.

34. Dinckan A, Gurkan A, Ozkan O, Dinc B, Yuksel Y, Akgul N, et al. Hepatic artery reconstruction with gonodal vein interposition: firs case in patients receiving liver from the living donor. Am J Case Rep. 2012;13:195-7.

35. Sirbu Boeti MP, Brasoveanu V, Shoaib S, Popescu I. Arterial Anastomosis in Living Donor Liver Transplantation. In Liver Transplantation - Technical Issues and Complications. Edited by Prof. Hesham Abdeldayem. Ed Intech. 2012; p 43-64.

36. Addeo P, Oussoultzoglou E, Fuchshuber P, Rosso E, Nobili C, Langella $S$, et al. Safety and outcome of combined liver and pancreatic resections. Br J Surg 2014 May;101(6):693-700.

37. Tran TB, Dua MM, Spain DA, Visser BC, Norton JA, Poultsides GA. Hepato-pancreatectomy: how morbid? Results from the national surgical quality improvement project. HPB (Oxford) 2015 Sep; 17(9): 763-9. 